Résumés des conférences et travaux

\title{
Archives orales et histoire des organisations
}

\section{Florence Descamps}

\section{OpenEdition \\ Journals}

\section{Édition électronique}

URL : https://journals.openedition.org/ashp/3169

DOI : $10.4000 /$ ashp.3169

ISSN : 1969-6310

Éditeur

Publications de l'École Pratique des Hautes Études

\section{Édition imprimée}

Date de publication : 1 septembre 2019

Pagination : 349-352

ISSN : 0766-0677

Référence électronique

Florence Descamps, "Archives orales et histoire des organisations », Annuaire de l'École pratique des hautes études (EPHE), Section des sciences historiques et philologiques [En ligne], 150 | 2019, mis en ligne le 12 juin 2019, consulté le 06 juillet 2021. URL : http://journals.openedition.org/ashp/3169 DOI : https://doi.org/10.4000/ashp.3169 


\title{
ARCHIVES ORALES ET HISTOIRE DES ORGANISATIONS
}

\author{
Maître de conférences : $\mathrm{M}^{\mathrm{me}}$ Florence DEsCAMPS
}

Programme de l'année 2017-2018 : I. Des archives sonores à la mémoire orale : la « fabrique » d'un patrimoine culturel immatériel? - II. Vertus heuristiques et apports épistémologiques des archives orales. - III. Histoire orale du ministère des Finances dans les années 1980: d'une alternance à l'autre, néolibéralisme ou social-libéralisme à la française?

L'année est divisée en trois trimestres.

\section{Des archives sonores à la mémoire orale : la «fabrique » d'un patrimoine culturel immatériel?}

L'articulation qui peut exister entre oralité, histoire, mémoire et patrimoine a fait en France l'objet de questionnements précoces de la part des sciences humaines et sociales; elle a donné lieu à l'invention de nouvelles pratiques d'enquête, appuyées sur des innovations technologiques en matière d'enregistrement sonore et de captation d'images. Le premier axe d'étude consiste donc à établir la généalogie et l'histoire de ces pratiques, depuis les premières fixations scientifiques de la parole du début du $\mathrm{Xx}^{\mathrm{e}}$ siècle jusqu'aux actuelles enquêtes de mémoire orale filmées. Une attention particulière est portée à la terminologie utilisée, de façon à la contextualiser et à l'historiciser : archives de la parole, traditions orales, enquêtes orales, histoire orale, archives orales, témoignages oraux, corpus oraux, mémoire orale, histoire visuelle, etc.

Le deuxième axe consiste à étudier comment ces pratiques d'enquête se sont doublées (ou non) de pratiques de patrimonialisation. Depuis les Archives de la Parole créées en 1911 au sein de la Sorbonne jusqu'à la notion de «patrimoine culturel immatériel », élaborée puis imposée par l'Unesco en 2003, le séminaire s'interroge sur la manière dont la parole enregistrée et la mémoire mise en parole se trouvent elles aussi prises dans la «fabrique » du patrimoine (N. Heinich). En quels termes et par quels canaux la demande de mémoire s'exprime-t-elle? Quels sont les liens entre mémoire individuelle et mémoire collective? Les archives orales permettent-elles de saisir une mémoire collective? Peut-on parler de patrimoine oral? Les sources orales font-elles ou non partie du patrimoine culturel immatériel? Si oui, pourquoi? Si non, à quelles conditions pourraient-elles y entrer?

Le troisième axe est délibérément pratique et méthodologique; il s'inscrit délibérément dans une démarche d'ingénierie et souhaite offrir aux auditeurs, chercheurs et professionnels des archives ou de la documentation les outils méthodologiques et scientifiques nécessaires pour constituer des corpus de données réutilisables par la recherche (définition d'une problématique, mise en série, accessibilité, vérifiabilité, accessibilité, documentation, citabilité). La question de l'adjonction de l'image animée est systématiquement discutée. Pourquoi filmer les entretiens? Quels sont les 
effets de tels ou tels dispositifs filmiques? Comment analyser les images produites? Quels sont les apports comparés de l'image et du son à l'entretien? L'image accélère-t-elle ou modifie-elle le processus de patrimonialisation? La problématique juridique et éthique qui s'impose désormais pour l'utilisation des données personnelles fait l'objet d'une séance entière. Pour appuyer cette réflexion, plusieurs études de cas ont été présentées, notamment les travaux de l'ethnologue Karin Le Petit au sein de l'Ethnopôle. La Fabrique des patrimoines en Normandie sur « les travailleurs de la mer »; la question des méthodes d'entretien comparées entre histoire, ethnographie et anthropologie a été spécifiquement posée. L'autre cas étudié a porté sur la constitution d'un corpus de témoignages oraux d'histoire sociale, ouvrière et industrielle, Usines en bord de Seine, au sein du Parc naturel des boucles de la Seine, par Marine Simon, doctorante en histoire.

\section{Vertus heuristiques et apports épistémologiques des archives orales}

Le deuxième trimestre a été consacré à un bilan des archives orales, trente-cinq ans après le congrès international d'histoire orale d'Aix en Provence, organisé par JeanClaude Bouvier et Philippe Joutard avec l'Association internationale d'histoire orale. La participation de docteurs ou de doctorants utilisant tous à un degré ou à un autre dans leur travail la source orale a permis d'ancrer ce bilan dans des travaux empiriques récents et innovants (A. Georges-Desvignes sur les grandes réformes universitaires Faure et Savary, M. Mériaux sur les sources et la transmission de l'ethnobotanique bretonne, B. Girault sur les «mémoires du ministère de l'Éducation nationale », J.-P. Legois sur « les mémoires étudiants des années $68 »)$. Nous avons pu dégager plusieurs thématiques qui seront retravaillées l'année prochaine : premièrement une typologie des usages scientifiques de la source orale, qui se donne à voir dans la structure matérielle du manuscrit (notes de bas de page, annexes, introduction, corps du texte, etc.). Deuxièmement, l'enjeu de la « visite » ou du réexamen des corpus oraux créés et archivisés à partir des années 1970 à l'intention « de l'historien du futur » ${ }^{1}$ et leur mise à l'épreuve en matière épistémologique. À cet égard, nous proposons quatre grands référentiels ou répertoires d'analyse qui peuvent permettre de saisir l'épaisseur polysémique des témoignages oraux et de distinguer plusieurs registres de compréhension et d'interprétation : un référentiel que nous appelons réaliste (positiviste) qui s'intéresse aux faits rapportés par le témoin; un référentiel discursif ou narratif qui s'intéresse au récit mis en œuvre par le témoin, ainsi qu'à l'oralité; un référentiel mémoriel qui s'intéresse aux formes de la ressouvenance chez le témoin; enfin, un référentiel documentaire qui s'intéresse au traitement des entretiens, à leur structuration et à la production des connaissances qui en découle. Notre troisième axe de réflexion s'attache plus précisément au référentiel mémoriel et cherche à préciser la définition du terme " régime de mémorialité », dérivé du " régime d'historicité »

1. G. Thuillier, «Pour la création d'archives orales », La Gazette des archives, no 92 (1976). p. 28-32; D. Schnapper et D. Hanet, "Archives orales et histoire des institutions sociales », Revue française de sociologie, 19-2 (1978), p. 261-275; «D’Hérodote au magnétophone : sources orales et archives orales », Les Annales. Économie, Sociétés, Civilisations, nº 1 (1980), p. 183-199. 
forgé par F. Hartog ${ }^{2}$ et utilisé par D. Peschanski depuis 2012 pour l'héritage mémoriel de la Seconde Guerre mondiale ${ }^{3}$. En quoi ce concept est-il opératoire pour les corpus d'archives orales constitués dans la seconde moitié du $\mathrm{xx}^{\mathrm{e}}$ siècle? Enfin, le quatrième axe de réflexion est celui de la valorisation des témoignages oraux, des médiations qu'on peut leur appliquer et de leur réécriture. Cette question a été abordée à l'occasion de la parution de l'ouvrage de E. Lengereau, historien des politiques publiques en architecture, auteur de l'ouvrage Architecture, urbanisme et pratiques de l'État, 1960-2010, Paris, La Documentation française, 2017.

\section{Histoire orale du ministère des Finances dans les années 1980 : d'une alternance à l'autre, néolibéralisme ou social-libéralisme à la française?}

Le troisième trimestre est consacré à l'histoire orale du ministère des Finances dans le dernier quart du $\mathrm{XX}^{\mathrm{e}}$ siècle. Le séminaire organise la confrontation des points de vue entre chercheurs et témoins, entre historiens de l'économie, historiens de l'administration, historiens du politique, économistes et politistes, en croisant sources écrites et sources orales. Le questionnement porte sur la contribution des hauts fonctionnaires du ministère des Finances à l'élaboration, à la décision et à l'application des successives politiques économiques et financières menées en France depuis 1981. La perception par les différentes directions du ministère des enjeux économiques et financiers auxquels a été confrontée la France, qu'ils soient nationaux ou internationaux, la culture politique, économique et technique des dirigeants administratifs, la capacité des services à élaborer des solutions en réponse aux problèmes mis à l'agenda, le choix des dispositifs administratifs destinés à la mise en œuvre des politiques publiques concernées, le face-à-face décideur-expert, le rôle des « entourages » et la chronologie fine du processus décisionnel font l'objet d'une attention particulière.

Depuis 2013, le séminaire explore le premier septennat de François Mitterrand. Questionnant les ruptures et les continuités communément admises, le séminaire s'efforce d'établir une chronologie fine du changement au ministère des Finances, tout en l'insérant dans les perspectives de longue durée qui marquent l'histoire économique de la France depuis la Ve République (ouverture européenne, libéralisation économique). L'étude de la décennie 1981-1992 a permis de dégager, au gré des alternances gouvernementales, des séquences historiques contrastées, soit politiquement mises en scène $(1981,1983,1986)$ soit au contraire minimisées ou rendues discrètes $(1982$, 1984), qui rendent compte par-delà les «émotions politiques » des continuités des politiques économiques et financières françaises depuis 1976 (cf. l'analyse par Laure Quennouëlle-Corre des figures de trois ministres de l'Économie et des finances : « les trois B » : Raymond Barre (1976-1981), Pierre Bérégovoy (1984-1986) et Édouard Balladur (1986-1988). Le séminaire est revenu sur le «tournant de la rigueur » de mars 1983 et son processus décisionnel (avec François Monier, conseiller de Pierre Mauroy, Louis Gallois, directeur de cabinet de J.-P. Chevènement, Michel Camdessus, directeur du Trésor), ainsi que sur l'expérience Balladur (avec Charles de Croisset,

2. Régimes d'historicité. Présentisme et expériences du temps, Paris, Le Seuil, 2003.

3. D. Peschanski, Les années noires : 1938-1944, Paris, Herman, 2012. 
directeur de cabinet, Jérôme Périer, biographe d'Alain Madelin, ministre de l'Industrie). Les travaux du séminaire ont été valorisés en 2018 dans un dossier thématique de la revue Vingtième siècle : « 1983, un tournant libéral? », nº 138, avril-juin 2018, p. 4-107.

De façon expérimentale, un second axe de recherche a été ajouté, sous le titre suivant: «Les femmes qui comptent». Ce programme, placé sous la direction de Laure Quennouëlle-Corre (CNRS) et de Sabine Effosse (université Paris-Nanterre), comporte trois volets d'étude : les femmes et l'argent dans leur vie privée, au sein de leur ménage ou dans leur vie professionnelle (entreprise, commerce, atelier); les femmes qui exercent des fonctions comptables et financières derrière le guichet, dans les banques et les institutions financières privées ou publiques; les femmes qui ont atteint les postes d'encadrement supérieurs ou de dirigeantes dans ces mêmes institutions. L'étude des femmes cadres au ministère des Finances nous a semblé pouvoir entrer dans ce programme, d'autant plus que nous avions déjà consacré à cette question un article en 2013 ( L'histoire des femmes cadres aux Finances 1939-1981: un plafond de plomb », Revue française d'administration publique, $\mathrm{n}^{\circ} 145,2013$, p. 39-63). Quatre anciennes administratrices des Finances ayant percé «le plafond de verre » ont fait l'objet d'entretiens biographiques approfondis, ce qui a permis de dégager des axes d'étude pour développer l'étude des femmes cadres dirigeantes aux Finances l'année prochaine. 